\title{
JOINT OPTIMIZATION OF RELAY POSITION AND POWER ALLOCATION IN COOPERATIVE BROADCAST WIRELESS NETWORKS
}

\author{
Ying Jin, Yimin D. Zhang, and Batu K. Chalise \\ Center for Advanced Communications, Villanova University, Villanova, PA 19085, USA
}

\begin{abstract}
In this paper, we examine the resource optimization problem in a broadcast relay network where a source broadcasts signals to the user receivers, which are distributed over a service region. The source does not have direct line-of-sight to the service area and the information is delivered through a relay. The objective of this paper is to jointly optimize the relay position as well as the power allocation between the source and relay so that the outage probability of the signal received at the user nodes is minimized. Two different optimization criteria, which respectively minimize the worst-case outage probability and the average outage probability, are used. The analyses are verified by simulation results.
\end{abstract}

Index Terms - Cooperative communications, broadcast network, joint optimization, resource allocation, outage probability

\section{INTRODUCTION}

Broadcasting signals to multiple user nodes that are distributed over a service region is of significant importance in various wireless communications applications. In particular, there is a class of broadcast networks where the source node does not have reliable wireless links to the user nodes and, therefore, it becomes necessary to exploit cooperative relays to assist the broadcast communications [1,2]. While cooperative communications have found wide applications in various commercial as well as military operations for the purpose from improving the communication reliability to extending service coverage areas [3, 4], our primary interest in this paper lies in unmanned aerial vehicle (UAV) networks that provide relay function to ground operations [5]. In particular, low-cost UAV swarms, in addition to their increasingly important roles in supporting reconnaissance, border patrol, traffic control, forest fire monitoring, and distributed irrigation control $[6,7]$, have also been found powerful in relaying information in an environment where the line-of-sight (LOS) between the source and the user nodes is obstructed due to terrestrial or urban structure obstructions. The objective of relaying in this case is to maintain reliable wireless communication links between the source and user nodes [8]. In such applications, the information may be targeted to a group of receivers, e.g., a team of soldiers on ground, and a broadcast relay wireless network is considered effective [1,2]. Because of the power limitation at the source (which is usually a portable device) and the relay (which is a small UAV in this case), an important objective in such a network is to minimize the outage probability with a low level of overall power transmitted from the source and relay nodes.

In this paper, we consider a broadcast relay network where a source broadcasts signals, through a relay, to a group of user receivers which are distributed over a circular service region. The source, relay, and each user node is assumed to exploit a single antenna. The decode-and-forward (DF) scheme is applied in the relay. The source and the user nodes are placed on ground and, due to LOS obstruction, the direct links between the source and the user nodes are considered highly attenuated and are thus negligible. On the other hand, the relay node is positioned with certain height above ground to achieve high link quality to both source and user nodes, whereas its position in other dimensions are to be optimized. The source-relay link as well as the relay-user links are assumed to be Rayleigh channels with their path attenuation being determined by their respective distance between the transmitter and the receiver. The objective of this paper is to jointly optimize the relay position as well as the power allocation between the source and relay so that the outage probability of the signal received at the user nodes is minimized. Two different optimization criteria, which respectively minimize the worst-case outage probability and the average outage probability, are considered. The optimized results are verified using simulation results.

Notations: We use lower-case (upper-case) bold characters to denote vectors (matrices). In particular, $\mathbf{I}_{N}$ denotes the $N \times N$ identity matrix. (. $)^{T}$ denotes the transpose of a matrix or vector, $\|\cdot\|$ denotes the Euclidean norm of a vector, and $\operatorname{Pr}(\cdot)$ denotes the probability operator.

\section{SYSTEM MODEL}

Consider a broadcast wireless network, depicted in Fig. 1, where $M$ user nodes, $U_{1}, \ldots, U_{M}$, are uniformly and independently distributed within a circular area of radius $L$. The source terminal is located at a distance of $R_{0}$ from the center of the service area which is set as the origin of the coordinate system. The source and all the user nodes are placed on the ground. Due to LOS obstruction due to, for example, terrain or urban structures, the direct links between the source and the user nodes are considered highly attenuated and thus are negligible.

Both polar and cartesian coordinate systems are used in this paper for the convenience of analysis. In both coordinate systems, we set the center of the circular service area as the coordinate origin. In the polar coordinate system, the direction of the source is set to be zero. The cartesian coordinate system can be correspondingly defined, where the $x$-axis coincides with the direction of the source node, and the $z$-axis points to the the upward elevation direction. The joint probability density function (pdf) of user node $U_{i}$, whose distance from the center of the circular service area is $l_{U_{i}} \in[0, L]$ and its spatial angle is $\theta_{U_{i}} \in[0,2 \pi)$, is described as $f\left(l_{U_{i}}, \theta_{U_{i}}\right)=l_{U_{i}} /\left(\pi L^{2}\right)$. The path loss corresponding to a pair of transmitter and receiver separated by distance $l$ is assumed to be $l^{\eta}$, where $\eta$ is the path loss exponent. In this paper, we assume $\eta=2$ for 


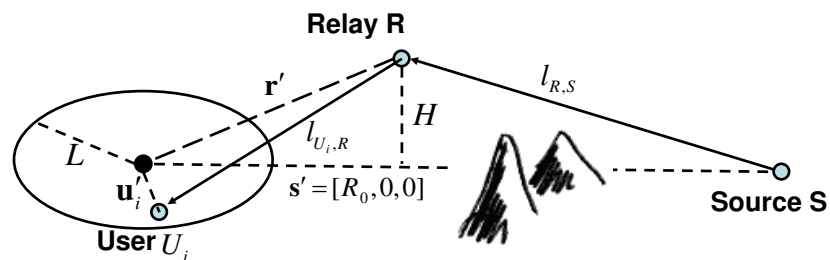

Fig. 1. Illustration of the network geometry.

the convenience of analysis. The fast fading of the channels is characterized as a Rayleigh channel with a unit variance. The relay and each user node have additive zero-mean circular symmetric complex white Gaussian noise with an identical variance of $\sigma_{n}^{2}$. Signal and noise are uncorrelated to each other.

We consider a relay $R$ whose altitude is set to be $H$. In practice, the relay altitude should be determined by the type of relay and the operation environment such that it is as low as possible to reduce the path loss whereas sufficiently high to avoid significant obstructions in both the source-relay and relay-user links. In this paper, $H$ is assumed to be fixed, and the optimization of the relay position focuses, therefore, on the two horizontal dimensions. We adopt the DF scheme at the relay, and two time slots are used in the relay protocol. In the first time slot, the source terminal transmits a normalized signal waveform $x(t)$ with a transmit power $\rho_{S}$ to the relay. After the relay node successfully decodes the received signal, it broadcasts the decoded signal in the second time slot to the user nodes with a power $\rho_{R}$. Denote $\rho=\rho_{S}+\rho_{R}$ as the total power transmitted from both the source and the relay. We introduce a variable $\nu, 0<\nu<1$, to represent the portion of total transmit power designated to the source, i.e., $\rho_{S}=\nu \rho$. The power transmitted at the relay, therefore, is denoted as $\rho_{R}=(1-\nu) \rho$. Given the total transmit power $\rho$, therefore, the optimization of the power allocation between the source and the relay nodes becomes the determination of the optimal value of $\nu$.

The signal received at the relay node is expressed as

$$
y_{R, S}(t)=\sqrt{\frac{\rho_{S}}{l_{R, S}^{2}}} h_{R, S}(t) s(t)+n_{R}(t),
$$

where $s(t)$ is the source signal, $h_{R, S}(t)$ and $l_{R, S}$ are, respectively, the time-varying Rayleigh fading channel and the distance between the source and the relay, and $n_{R}(t) \sim \mathcal{N C}\left(0, \sigma_{n}^{2}\right)$ is the additive relay noise. The channel coefficient $h_{R, S}(t)$ is assumed to have unit variance, i.e., $h_{R, S}(t) \sim \mathcal{N C}(0,1)$.

The instantaneous receive signal-to-noise ratio (SNR) at the relay is obtained from (1) as $\gamma_{R, S}(t)=\rho_{S}\left|h_{R, S}(t)\right|^{2} /\left(l_{R, S}^{2} \sigma_{n}^{2}\right)$, which follows an exponential distribution with parameter $\lambda_{R, S}=$ $\left(l_{R, S}^{2} \sigma_{n}^{2}\right) / \rho_{S}$. The outage probability at the relay is defined as the probability that the capacity of the channel between the source and the relay, $\frac{1}{2} \log _{2}\left(1+\gamma_{R, S}\right)$, is smaller than a predetermined data rate requirement, denoted as $\alpha$. The factor $1 / 2$ is used to emphasize the half-duplex nature of the relay. Equivalently, the outage probability can be expressed as the probability that the received SNR is below a corresponding threshold $\gamma_{0}=2^{2 \alpha}-1$. On the other hand, when the received SNR is higher than the threshold $\gamma_{0}$, the user is assumed to be able to decode the received message with a negligible probability of error [2]. Therefore, the outage probability from the source to the relay node is expressed as

$$
P_{R, S}=\operatorname{Pr}\left[\frac{1}{2} \log _{2}\left(1+\gamma_{R, S}\right)<\alpha\right]=1-\exp \left(-\lambda_{R, S} \gamma_{0}\right) .
$$

At the second time slot, the relay broadcasts the signal $s(t)$ to the user nodes only if it correctly decodes the signal. The signal received at user node $U_{i}$ becomes

$$
y_{U_{i}, R}(t)=\sqrt{\frac{\rho_{R}}{l_{U_{i}, R}^{2}}} h_{U_{i}, R}(t) s(t)+n_{U_{i}}(t),
$$

where $n_{U_{i}}(t) \sim \mathcal{N C}\left(0, \sigma_{n}^{2}\right)$ is the receiver noise.

The outage probability from the relay to user node $U_{i}, i=$ $1, \ldots, M$, is

$$
P_{U_{i}, R}=1-\exp \left(-\lambda_{U_{i}, R} \gamma_{0}\right),
$$

where $\lambda_{U_{i}, R}=\left(l_{U_{i}, R}^{2} \sigma_{n}^{2}\right) / \rho_{R}$.

Because of the absence of the direct path between the source and the user nodes, a user node can successfully recover the signal transmitted from the source only when both the source-relay and the relay-user links are successfully decoded. Therefore, the outage probability can be expressed as

$$
\begin{aligned}
P_{U_{i}}^{Q} & =1-\left(1-P_{R, S}\right)\left(1-P_{U_{i}, R}\right) \\
& =P_{R, S}+P_{U_{i}, R}-P_{R, S} P_{U_{i}, R} .
\end{aligned}
$$

Considering a practical situation where $P_{R, S}$ and/or $P_{U_{i}, R}$ take low values, we can ignore the term $P_{R, S} P_{U_{i}, R}$, yielding the following approximation

$$
P_{U_{i}}^{Q} \approx P_{R, S}+P_{U_{i}, R}
$$

Applying the results of (2) and (4), the first-order Taylor expansion of the above expression leads to the following approximation

$$
P_{U_{i}}^{Q} \approx \lambda_{R, S} \gamma_{0}+\lambda_{U_{i}, R} \gamma_{0}=\frac{\gamma_{0} l_{R, S}^{2} \sigma_{n}^{2}}{\rho_{s}}+\frac{\gamma_{0} l_{U_{i}, R}^{2} \sigma_{n}^{2}}{\rho_{R}} .
$$

Let three-dimensional (3-D) position vectors of the source, relay, and user node $U_{i}$ be, respectively, denoted as $\mathbf{s}^{\prime}=\left[x_{S}, y_{S}, 0\right]^{T}$, $\mathbf{r}^{\prime}=\left[x_{R}, y_{R}, H\right]^{T}$, and $\mathbf{u}_{i}^{\prime}=\left[x_{U_{i}}, y_{U_{i}}, 0\right]^{T}$, whereas $\mathbf{s}=$ $\left[x_{S}, y_{S}\right]^{T}, \mathbf{r}=\left[x_{R}, y_{R}\right]^{T}$, and $\mathbf{u}_{i}=\left[x_{U_{i}}, y_{U_{i}}\right]^{T}$ are their corresponding projections to the two-dimensional (2-D) horizontal plain. Then, the above expression becomes

$$
\begin{aligned}
P_{U_{i}}^{Q} & \approx \frac{\gamma_{0} \sigma_{n}^{2}}{\rho}\left(\frac{1}{\nu} l_{R, S}^{2}+\frac{1}{1-\nu} l_{U_{i}, R}^{2}\right) \\
& =\frac{\gamma_{0} \sigma_{n}^{2}}{\rho}\left(\frac{1}{\nu}\|\mathbf{r}-\mathbf{s}\|^{2}+\frac{1}{1-\nu}\left\|\mathbf{u}_{i}-\mathbf{r}\right\|^{2}+\frac{H^{2}}{\nu(1-\nu)}\right) .
\end{aligned}
$$

\section{OPTIMIZATION BASED ON WORST-CASE OUTAGE PROBABILITY}

We first consider the optimization of the relay position and power allocation that minimize the worst-case outage probability. This ensures the quality of service of all user nodes within the service area.

The optimization problem is expressed as

$$
\min _{\mathbf{r}, \nu} \sup \left\{P_{U_{i}}^{Q} \mid\left\|\mathbf{u}_{i}\right\| \leq L\right\}
$$

Utilizing (8), this expression can be rewritten as the following minmax problem

$$
\begin{aligned}
& \min _{\mathbf{r}, \nu} \max _{\mathbf{u}_{i}}\left(\frac{1}{\nu}\|\mathbf{r}-\mathbf{s}\|^{2}+\frac{1}{1-\nu}\left\|\mathbf{u}_{i}-\mathbf{r}\right\|^{2}+\frac{H^{2}}{\nu(1-\nu)}\right) \\
& \text { s.t. }\left\|\mathbf{u}_{i}\right\| \leq L .
\end{aligned}
$$


Note that the constant terms $\gamma_{0} \sigma_{n}^{2} / \rho$ included in the outage probability is omitted in the above cost function for notational simplicity. We first solve the inner maximization problem. In this case, we rewrite it as a minimization objective function using Lagrange method

$$
\begin{aligned}
f\left(\mathbf{u}_{i}, \nu, \lambda\right)= & -\left(\frac{1}{\nu}\|\mathbf{r}-\mathbf{s}\|^{2}+\frac{1}{1-\nu}\left\|\mathbf{u}_{i}-\mathbf{r}\right\|^{2}+\frac{1}{\nu(1-\nu)} H^{2}\right) \\
& +\lambda\left(\mathbf{u}_{i}^{T} \mathbf{u}_{i}-L^{2}\right)
\end{aligned}
$$

where $\lambda \geq 0$ is the Lagrange multiplier. The partial derivative of $f$ with respect to $\mathbf{u}_{i}$ is obtained as

$$
\frac{\partial f}{\partial \mathbf{u}_{i}}=-\frac{2}{(1-\nu)}\left(\mathbf{u}_{i}-\mathbf{r}\right)+2 \lambda \mathbf{u}_{i}
$$

and the corresponding Hessian matrix is

$$
\mathbf{H}(f)=\frac{\partial^{2} f}{\partial \mathbf{u}_{i} \partial \mathbf{u}_{i}^{T}}=\left[-\frac{2}{(1-\nu)}+2 \lambda\right] \mathbf{I}_{2} .
$$

Setting the partial derivative to be zero yields the relationship between the worst-case user position $\mathbf{u}_{i}$ and the relay position $\mathbf{r}$ as $\mathbf{u}_{i}=\mathbf{r} /[1-(1-\nu) \lambda]$. To eliminate the uncertainty due to $\nu$ and $\lambda$, we further consider the complementary slackness, i.e., $\lambda\left(\mathbf{u}_{i}^{T} \mathbf{u}_{i}-\right.$ $\left.L^{2}\right)=0$. It is clear that $\lambda=0$ is not a suited solution because it yields a negative-definite Hessian matrix (and the corresponding solution is $\mathbf{u}_{i}=\mathbf{r}$ which is the best-case user position in the relay-user link). Thus, we conclude that $\lambda \neq 0$, which implies $\mathbf{u}_{i}= \pm L \mathbf{r} /\|\mathbf{r}\|$. Among these two solutions, $\mathbf{u}_{i}=-L \mathbf{r} /\|\mathbf{r}\|$ is determined to be the worst-case user position because it yields a positive definite Hessian matrix and corresponds to a longer distance to the relay than the other position $L \mathbf{r} /\|\mathbf{r}\|$ does. Substituting this result back to (10), we are ready to solve the minimization problem with respect to $\mathbf{r}$. The objective function becomes

$$
\begin{aligned}
g(\mathbf{r}, \nu) & =\frac{1}{\nu}\|\mathbf{r}-\mathbf{s}\|^{2}+\frac{1}{1-\nu}\left\|-\frac{\mathbf{r}}{\|\mathbf{r}\|} L-\mathbf{r}\right\|^{2}+\frac{1}{\nu(1-\nu)} H^{2} \\
& =\frac{1}{\nu}\|\mathbf{r}-\mathbf{s}\|^{2}+\frac{1}{1-\nu}(L+\|\mathbf{r}\|)^{2}+\frac{1}{\nu(1-\nu)} H^{2}
\end{aligned}
$$

Setting the partial derivative of $g$ with respect to $\mathbf{r}$ to be zero, we obtain

$$
\mathbf{r}+\nu L \frac{\mathbf{r}}{\|\mathbf{r}\|}=(1-\nu) \mathbf{s}
$$

From (15), it is clear that the vectors $\mathbf{r}, \mathbf{u}_{\mathbf{i}}$ and $\mathbf{s}$ are collinear, implying $y_{R}=y_{U_{i}}=0$. Therefore, we can denote $\mathbf{s}^{\prime}=\left[R_{0}, 0,0\right]$, $\mathbf{r}^{\prime}=\left[x_{R}, 0, H\right]$. From (15), we obtain $x_{R}=(1-x) R_{0}-x L$, and the worst-case user position is $\mathbf{u}_{i}^{\prime}=[-L, 0,0]$. That is, the worst-case optimization problem is equivalent to a point-to-point relay problem with the user node located at $\mathbf{u}_{i}^{\prime}=[-L, 0,0]$. The objective function $g$ can now be written as

$$
\begin{aligned}
g(\nu) & =\frac{1}{\nu}\left(x_{R}-R_{0}\right)^{2}+\frac{1}{1-\nu}\left(-L-x_{R}\right)^{2}+\frac{1}{\nu(1-\nu)} H^{2} \\
& =\left(R_{0}+L\right)^{2}+\frac{1}{\nu(1-\nu)} H^{2} .
\end{aligned}
$$

It is clear from this expression that the optimal value of $\nu$ is $\nu=1 / 2$, which means equal transmit power allocation between the source and the relay nodes. The best relay position is $\mathbf{r}^{\prime}=\left[\frac{1}{2}\left(R_{0}-L\right), 0, H\right]$, i.e., its projection to the 2-D ground plane lies in the mid-point between the source and the worst-case user node. The corresponding outage probability at the worst-case position within the service area, under the optimum relay positioning and power allocation, is expressed as

$$
P_{\min }^{\mathrm{worst}}=\frac{\gamma_{0} \sigma_{n}^{2}}{\rho}\left[\left(R_{0}+L\right)^{2}+4 H^{2}\right]
$$

\section{OPTIMIZATION BASED ON AVERAGE OUTAGE PROBABILITY}

In this section, we examine the relay positioning and transmit power allocation using the average outage probability as the optimization criterion, i.e., the objective is to minimize the outage probability averaged over all user nodes distributed in the circular service area.

It can be easily verified that, due to symmetry, the optimum relay position should lie on top of the $x$-axis, i.e., $y_{R}=0$. To show this, we consider the outage probability of four symmetric nodes with 2-D coordinates $\mathbf{u}_{i 1}=\left(x_{\mathbf{u}_{i}}, y_{\mathbf{u}_{i}}\right), \mathbf{u}_{i 2}=\left(x_{\mathbf{u}_{i}},-y_{\mathbf{u}_{i}}\right), \mathbf{u}_{i 3}=$ $\left(-x_{\mathbf{u}_{i}},-y_{\mathbf{u}_{i}}\right)$ and $\mathbf{u}_{i 4}=\left(-x_{\mathbf{u}_{i}}, y_{\mathbf{u}_{i}}\right)$. The sum outage probability is expressed as

$$
\begin{aligned}
Q & =P_{U_{i 1}}^{Q}+P_{U_{i 2}}^{Q}+P_{U_{i 3}}^{Q}+P_{U_{i 4}}^{Q} \\
& =\sum_{k=1}^{4} \frac{\gamma_{0} \sigma_{n}^{2}}{\rho}\left(\frac{1}{\nu}\|\mathbf{r}-\mathbf{s}\|^{2}+\frac{1}{1-\nu}\left\|\mathbf{u}_{i k}-\mathbf{r}\right\|^{2}+\frac{1}{\nu(1-\nu)} H^{2}\right) \\
& =\frac{\gamma_{0} \sigma_{n}^{2}}{\rho}\left(\frac{4}{\nu}\|\mathbf{r}-\mathbf{s}\|^{2}+\frac{1}{1-\nu} \sum_{k=1}^{4}\left\|\mathbf{u}_{i k}-\mathbf{r}\right\|^{2}+\frac{4}{\nu(1-\nu)} H^{2}\right) .
\end{aligned}
$$

Equating the partial derivative of $Q$ with respect to $\mathbf{r}$ to zero, we obtain $\mathbf{r}=(1-\nu) \mathbf{s}+(\nu / 4) \sum_{k=1}^{4} \mathbf{u}_{i k}=(1-\nu) \mathbf{s}$, which implies $\mathbf{r}^{\prime}=\left[(1-\nu) R_{0}, 0, H\right]^{T}$. The average outage probability is evaluated in the polar coordinate system as

$$
\begin{aligned}
P^{\mathrm{ave}}= & \int_{0}^{L} \int_{0}^{2 \pi} \frac{l_{U_{i}}}{\pi L^{2}} P_{U_{i}} d \theta d l_{U_{i}} \\
= & \int_{0}^{L} \int_{0}^{2 \pi} \frac{l_{U_{i}} \gamma_{0} \sigma_{n}^{2}}{\pi L^{2} \rho}\left(\frac{1}{\nu}\left\|\mathbf{r}^{\prime}-\mathbf{s}^{\prime}\right\|^{2}+\frac{1}{1-\nu}\left\|\mathbf{u}_{i}^{\prime}-\mathbf{r}^{\prime}\right\|^{2}\right) d \theta d l_{U_{i}} \\
= & \frac{\gamma_{0} \sigma_{n}^{2}}{\rho} \int_{0}^{L} \int_{0}^{2 \pi} \frac{l_{U_{i}}}{\pi L^{2}}\left[\frac{1}{\nu}\left(-\nu R_{0}\right)^{2}+\frac{1}{1-\nu}\left((1-\nu) R_{0}\right.\right. \\
& \left.\left.-l_{U_{i}} \cos (\theta)\right)^{2}+\frac{1}{1-\nu} l_{U_{i}}^{2} \sin ^{2}(\theta)+\frac{1}{\nu(1-\nu)} H^{2}\right] d \theta d l_{U_{i}} \\
= & \frac{\gamma_{0} \sigma_{n}^{2}}{\rho}\left(R_{0}^{2}+\frac{L^{2}}{2(1-\nu)}+\frac{H^{2}}{\nu(1-\nu)}\right) .
\end{aligned}
$$

The partial derivative of $P^{\text {ave }}$ with respect to $\nu$ is obtained as,

$$
\frac{\partial P^{\text {ave }}}{\partial \nu}=\frac{\gamma_{0} \sigma_{n}^{2}}{\rho} \frac{L^{2} 2 \nu(1-\nu)-\left(\nu L^{2}+2 H^{2}\right)(2-4 \nu)}{4 \nu^{2}(1-\nu)^{2}} .
$$

Equating this to zero yields (only the positive value is kept)

$$
\nu_{\mathrm{opt}}=\sqrt{4 \kappa^{4}+2 \kappa^{2}}-2 \kappa^{2},
$$

which is an increasing function of $\kappa$, defined as $\kappa=H / L$, and $\nu_{\mathrm{opt}}$ is upper bounded by 0.5 . The corresponding average outage probability is

$$
P_{\mathrm{min}}^{\mathrm{ave}}=\frac{\gamma_{0} \sigma_{n}^{2}}{\rho}\left(R_{0}^{2}+\frac{L^{2}}{2+8 \kappa^{2}-4 \sqrt{4 \kappa^{4}+2 \kappa^{2}}}\right) .
$$




\section{SIMULATION RESULTS}

In the simulations, the noise power is normalized to unity. The total transmit power is set to be $\rho=95 \mathrm{~dB}$, which yields the receive SNR to be above a threshold of $\gamma=4.78 \mathrm{~dB}$ at a distance of $1,000 \mathrm{~m}$. For each case, the outage probability results are shown as the average of 10 independent trials. Unless otherwise specified, the following default parameters are used: $L=150 \mathrm{~m}, R_{0}=1000 \mathrm{~m}, H=500 \mathrm{~m}$, and $N=100$ users are assumed in simulated results.

To demonstrate the validity and the applicability of the optimized results, the analytical results are compared in Fig. 2 with those simulated using $N=10,100$, and 1000 randomly distributed receive nodes in the service region with a uniform distribution. It is verified that the analytic result well coincides with the simulated results when 100 or more receive users are present. Nevertheless, the result is sufficiently close to the simulation results with only 10 users.

In Fig. 3, the worst-case and average outage probabilities are plotted with respect to the $x$-coordinate of the relay for different relay height. For each relay location, the power allocation factor $\nu$ is set to be respectively optimal. For each scenario, the respective analytic result is shown as a circle. It is seen that the outage probability degrades with a higher value of relay height due to longer propagation distance. The optimal relay position does not significantly change for the relay heights computed as they generally yield a large value of $\kappa$.

In Fig. 4, the outage probability is depicted for different values of the radius of the service region. The optimal relay position under the worst-case outage probability criterion changes accordingly, whereas that under the average outage probability criterion does not visibly change. Fig. 5 shows the outage probability with different source distances. Obviously, as the source moves closer to the service region, the relay position also becomes closer.

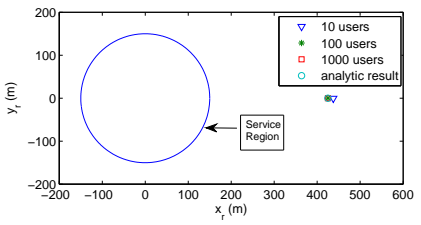

(a) Worst-case

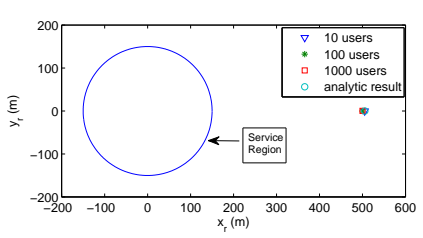

(b) Average
Fig. 2. Comparison of the analytical and simulated results of the optimal relay position.

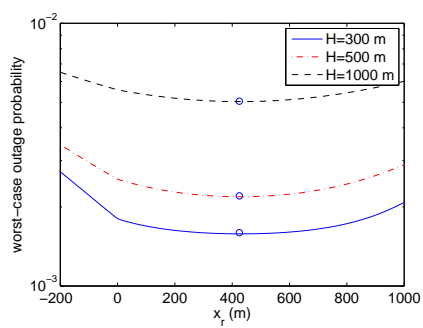

(a) Worst-case

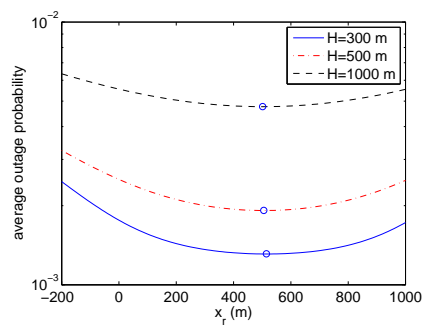

(b) Average
Fig. 3. Outage probability with different relay heights.

\section{CONCLUSIONS}

In this paper, a joint optimization problem of the the relay position as well as the transmit power allocation has been examined in a broad-

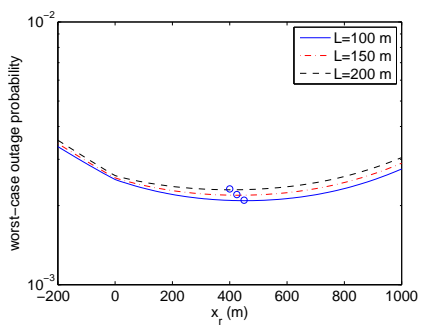

(a) Worst-case

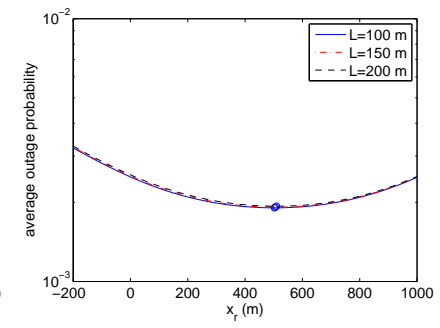

(b) Average
Fig. 4. Outage probability with different radius of the service region.

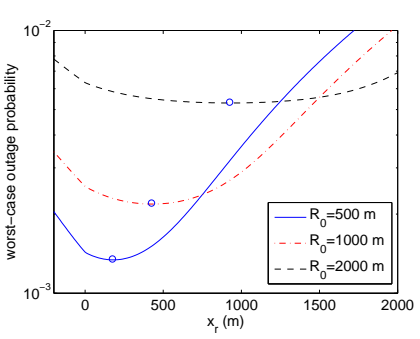

(a) Worst-case

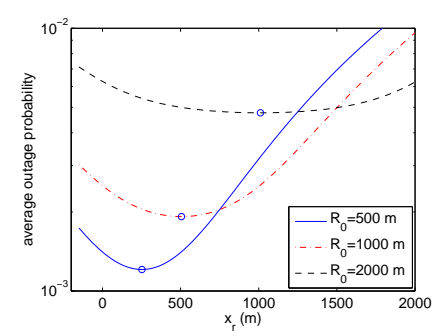

(b) Average
Fig. 5. Outage probability with different source distance.

cast relay network such that the receiver outage probability is minimized. Analytical solutions of the relay position and the transmit power allocation are derived under both the worst-case and the average outage probability criteria. The optimized results show good agreement with the simulation results with a moderate number of distributed receive nodes.

\section{REFERENCES}

[1] R. Zhang, C. C. Chai, Y. Liang, "Joint beamforming and power control for multiantenna relay broadcast channel with QoS constraints," IEEE Trans. Signal Proc., vol. 57, no. 2, pp. 726-737, 2009.

[2] H. V. Zhao and W. Su, "Distributed cooperative multicast in wireless networks: performance analysis and optimal power allocation," in Proc. IEEE ICASSP, pp. 3334-3337, Dallas, TX, March 2010.

[3] G. Kramer, I. Maric, and R. D. Yates, Cooperative Communications, Now Publishers, 2007.

[4] K. J. R. Liu, A. K. Sadek, W. Su, and A. Kwasinski, Cooperative Communications and Networking, Cambridge Univ. Press, 2008.

[5] X. Li and Y. D. Zhang, "Multi-source cooperative communications using multiple small relay UAVs," in Proc. IEEE Globecom Workshop on Wireless Networking for Unmanned Aerial Vehicles Program, Miami, FL, Dec. 2010.

[6] D. Casbeer, S. Li, R. Beard, T. McLain, and R. Mehra, "Forest fire monitoring with multiple small UAVs," in Proc. American Control Conf., Portland, OR, pp. 3530-3535, June 2005.

[7] H. Chao, M. Baumann, et al. , "Band-reconfigurable multi-UAVbased cooperative remote sensing for real-time water management and distributed irrigation control," in Proc. IFAC World Congress, Seoul, Korea, July 2008.

[8] P. Zhan, K. Yu, and A. Swindleurst, "Wireless relay communications using an unmanned aerial vehicle," in Proc. IEEE SPAWC, Cannes, France, July 2006. 